\title{
ALBUMINEMIA E TERAPIA MEDICAMENTOSA NO PROGNÓSTICO DE IDOSOS HOSPITALIZADOS
}

\author{
Fabiana Xavier Cartaxo Salgado ${ }^{1 *}$, Lucy Gomes Vianna ${ }^{2}$, Adriana Giavoni ${ }^{3}$, Gislane Ferreira de Melo ${ }^{4}$, Margô Gomes de Oliveira Karnikowski ${ }^{5}$
}

Trabalho realizado na Universidade de Brasília - UnB, Brasília, DF

*Correspondência:

QS 07 Rua 218 - Casa 52

Águas Claras

Brasília - DF

CEP: $71969-540$

Telefone: (61) 3356-8435

Fax: (61) 3263-5435

fabianacartaxo@yahoo.com.br

\section{RESUMO}

ОвJEтIvos. O presente estudo teve por objetivo investigar a relação entre os níveis de albumina sérica, medicamentos utilizados, tempo de internação e número de doenças com o prognóstico de indivíduos idosos hospitalizados.

Métodos. Estudo descritivo transversal e retrospectivo por meio de revisão de prontuários. Foram coletadas variáveis tais como: doença motivadora da internação, albuminemia, medicamentos utilizados e sua afinidade com a albumina e tempo de internação em uma população de idosos de um hospital público brasileiro. Foi utilizada a regressão múltipla para avaliar se a idade, dias de internação, medicamento com e sem afinidade a albumina, número de doenças motivadoras da internação foram preditores do óbito. A relação entre a variável dependente e as independentes foi analisada por Two-way ANOVA. Resultados. A amostra constituiu-se de 277 idosos, sendo 151 com hipoalbuminemia e sob uso de em média $6,6 \pm 3,80$ medicamentos diferentes cuja maioria apresentava afinidade pela albumina $(4,96$ $\pm 2,64)$. Os resultados demonstraram que o número de medicamentos com afinidade a albumina, a albuminemia e o período de internação foram preditores do óbito $(r=0.47 ; R 2=0.224),(F(8.170)$ $=6.13 ; p=0.001)$. A relação entre a variável dependente e as independentes resultou na equação matemática: (óbito= 0,545 - 0,374 (albuminemia) - 0,195 (dias de internação) + 0,175 (número de medicamentos com afinidade à albumina)).

Conclusão. A equação obtida para este estudo demonstrou que a albuminemia e os dias de internação foram inversamente correlacionados ao óbito e diretamente correlacionados com o elevado número de medicamentos ligados à albumina.

UnIteRmos: Idoso fragilizado. Hipoalbuminemia. Medicamentos sob prescrição.

\section{INTRODUÇÃO}

O envelhecimento populacional éfenômeno que merece destaque visto seu acentuado crescimento em todo o mundo ${ }^{1,2}$. No âmbito da saúde pública, o aumento da população idosa determina a modificação das necessidades por recursos capazes de proporcionar saúde, envolvendo medidas de prevenção ou intervenção com aporte a serviços médico-hospitalares e ao uso de medicamentos ${ }^{3,4}$.

0 processo de envelhecimento tem sido relatado na literatura como um fator contribuinte no aumento da demanda por serviços de saúde, havendo uma correlação com internações hospitalares mais frequentes e com tempo prolongado de ocupação do leito por idosos ${ }^{2}$. A maior prevalência de hospitalizações deste segmento etário está relacionada à presença de doenças crônicas não transmissíveis (DCNT) e a evolução da gravidade das doenças infecciosas.

Idosos internados com diagnóstico de desnutrição, processo inflamatório grave, insuficiência renal e hepática apresentam estreita relação com hipoalbuminemia ${ }^{5,6,7}$. Esta condição concorre para um prognóstico desfavorável com período de internação hospitalar prolongada e maior risco de complicações clínicas ${ }^{8,9}$. Em adição, idosos com hipoproteinemia podem não ser responsivos a medicação e podem apresentar alterações no efeito farmacológico quando se administra medicamentos com afinidade a proteínas plasmáticas ${ }^{8}$. A relevância da afirmação recai sobre o uso de medicamentos como a principal ferramenta terapêutica em ambiente nosocomial, sobretudo na população idosa, que utiliza de oito a 15 fármacos diferentes ${ }^{10,11,12}$. Cabe ressaltar que boa parte dos fármacos apresenta afinidade pelas proteínas séricas e que apenas a fração livre é responsável pelos efeitos farmacológicos. Assim, fármacos ácidos ligam-se à albumina e os básicos à $a 1$-glicoproteína ácida. Portanto, a presença de hipoalbuminemia em idosos pode induzir ao aumento na

1. Mestre em gerontologia pela Universidade Católica de Brasília e funcionária da Secretaria de Estado de Saúde do Distrito Federal, Brasília, DF

2. Doutora em fisiologia pela University of London-Inglaterra e pesquisadora do programa de pós-graduação Stricto sensu em gerontologia da Universidade Católica de Brasília, Brasília, DF

3. Doutora em Psicologia pela Universidade de Brasília e professora adjunta da Universidade Católica de Brasília, Brasília, DF

4. Doutora em Educação Física pela Universidade Católica de Brasília e professora adjunta da Universidade Católica de Brasília, Brasília, DF

5. Doutora em patologia molecular pela Universidade de Brasília e professora adjunta da Universidade de Brasília, Brasília, DF 
fração livre dos fármacos que se ligam à albumina induzindo a uma maior toxicidade ${ }^{13,14}$.

Estudos relatam proporção inversa entre os níveis de albumina com o tempo de internação hospitalar, morbidade e mortalidade, evidenciando o nível sérico de albumina como preditor de risco de morte ${ }^{15,16}$. A associação da morbi-mortalidade decorrente de complicações advindas da hipoalbuminemia pode incluir as alterações farmacocinéticas associadas ao uso de medicamento com afinidade por esta proteína.

Outro aspecto a ser considerado em idosos hospitalizados é o número e a gravidade das enfermidades, sendo que as doenças do aparelho circulatório e respiratório bem como as neoplasias, encontram-se diretamente relacionadas com a mortalidade em idosos ${ }^{17,18}$. Portanto, desnutrição, o número e a gravidade das doenças, os medicamentos administrados, o tempo de internação e a própria idade se constituem em importantes fatores a serem observados no que se refere ao prognóstico do paciente idoso hospitalizado.

O presente artigo propõe-se a investigar a relação entre os níveis de albumina sérica, medicamentos utilizados, tempo de internação e número de doenças com o prognóstico de indivíduos idosos hospitalizados.

\section{MÉTOdos}

Realizou-se estudo descritivo transversal e retrospectivo baseado na determinação do perfil qualitativo e quantitativo das prescrições de medicamentos utilizados pela população amostral. Consideraram-se como critérios de inclusões pacientes idosos internados na unidade de clínica médica de hospital público do Distrito Federal, com idade igual ou superior a 65 anos, de ambos os sexos, no período de setembro de 2007 a fevereiro de 2008. Foram coletados dados referentes ao sexo, à idade, aos medicamentos utilizados durante a internação, período de internação, o desfecho clínico, a doença motivadora da internação e albuminemia. Entre as doenças motivadoras da internação, foram consideradas como distúrbios nutricionais: gastrite, gastroenterite, gastroenterocolite, úlceras do trato digestivo, diarreia, enterorragia, hemorragia digestiva e esofagite. Entre as cardiopatias foram consideradas: insuficiência cardíaca congestiva, insuficiência aguda do miocárdio, miocardiopatia chagásica, insuficiência coronariana, derrame pericárdico e insuficiência aórtica.

Todos os dados foram obtidos pesquisando-se os prontuários médicos preenchidos durante o período de internação. Registrou-se a Denominação Comum Brasileira - DCB de cada medicamento e via de administração. Foram excluídos da pesquisa aqueles medicamentos considerados de urgência, ou seja, administradas esporadicamente para situações pontuais envolvendo risco iminente de morte ou dores agudas, assim como eletrólitos acrescentados a hidratação venosa e a insulina. Todos os demais medicamentos foram incluídos no banco de dados e analisados. Os princípios ativos contidos em cada produto foram agrupados por classes terapêuticas conforme 0 ATC/WHO ${ }^{19}$. As fontes utilizadas para investigação do grau de afinidade dos medicamentos à albumina, encontrados neste estudo foram Micromedex ${ }^{\circledR}$ Healthcare Series ${ }^{20}$, Base de datos del medicamento do Consejo General de Colegios Oficiales de Farmacéuticos ${ }^{21}$ e bulas individuais de alguns medicamentos.
Para um dos medicamentos não se encontrou a determinação da afinidade a albumina mesmo após investigação na literatura e solicitação deste dado junto ao laboratório fabricante.

Utilizou-se como critério de normalidade de albuminemia valores iguais ou superiores a $3,5 \mathrm{~g} / \mathrm{dl}^{22,23}$

As análises estatísticas foram realizadas empregando-se 0 pacote de programas SPSS versão 10.0 for Windows utilizandose as seguintes técnicas: teste $T$ para amostras independentes, One-way ANOVA, Two-way ANOVA e de regressão multivariada. Para estabelecer a relação entre o uso de medicamentos com ou sem afinidade pela albumina e os níveis sérios desta proteína foi utilizado a análise Two-way ANOVA. Foi utilizada a regressão múltipla para avaliar se as variáveis independentes tais como idade, dias de internação, albuminemia, medicamentos com e sem afinidade a albumina, número de doenças motivadoras da internação eram preditoras do óbito, que neste caso é a variável dependente.

Este estudo foi aprovado pelo Comitê de Ética sob nº 089/07 - Comitê de Ética em Pesquisa da Secretaria de Saúde do Distrito Federal - CEP/SES/DF com consentimento formal da chefia da unidade de saúde pesquisada.

\section{Resultados}

Características gerais da amostra

A amostra foi constituída por 277 pacientes idosos, com média de idade igual 75,97 $\pm 8,08$ anos, sendo 47,7\% ( $n=$ 132) do sexo feminino e $52,3 \%(n=145)$ do sexo masculino. Noventa e oito idosos (35,3\%) não apresentavam os níveis séricos de albumina determinados e dos 179 pacientes com solicitação da dosagem desta proteína, 84,3\% ( $n=151)$ possuíam hipoalbuminemia, $15,7 \%(n=28)$ idosos possuíam níveis normais de albumina sérica e nenhum paciente apresentou hiperalbuminemia. Dos idosos com albumina sérica determinada, $52,3 \%(n=79)$ eram do sexo masculino e $47,7 \%$ $(n=72)$ do sexo feminino.

O nível médio de albumina para os idosos com hipoalbuminemia foi de 2,74 $\pm 0,46 \mathrm{~g} / \mathrm{dL}$ e com albuminemia normal foi de 3,73 $\pm 0,22 \mathrm{~g} / \mathrm{dL}$, havendo diferença significativa entre as médias para os dois grupos [t(177) $=-11,27 ; p=0,001]$.

\section{Doenças motivadoras da internação}

As doenças motivadoras da internação mais frequentemente apresentadas foram as Pneumonias $(n=48)$, a infecção do trato urinário-ITU $(n=46)$, neoplasias $(n=42)$, distúrbios gástricos $(n=40)$, cardiopatias $(n=35)$, Acidente Vascular Encefálico AVE $(n=32)$, seguido de Doença Pulmonar Obstrutiva Crônica DPOC e Diabetes mellitus- DM, ambos com $n=22$ (Tabela 1 ).

Cabe ressaltar que 164 pacientes apresentavam mais de uma doença motivadora no momento da internação. Além das doenças que motivaram a internação foram estudadas as doenças crônicas não transmissíveis, presentes em 90,3\% $(n=250)$ da população investigada. A Hipertensão Arterial Sistêmica $(n=141)$ destacou-se como a mais prevalente, seguida do Diabetes mellitus ( $n=81)$, das Cardiopatias $(n=77)$, das neoplasias $(n=38)$, das Doenças Pulmonares Obstrutivas Crônicas $(n=35)$, das sequelas do Acidente Vascular Encefálico $(n=21)$ e da Doença de Chagas $(n=17)$.

$\mathrm{AVE}=$ acidente vascular encefálico; $\mathrm{DM}=$ Diabetes mellitus; 


\begin{tabular}{|c|c|c|c|}
\hline \multicolumn{2}{|c|}{$\begin{array}{l}\text { População total de idosos } \\
(\mathrm{n}=277)\end{array}$} & \multicolumn{2}{|c|}{$\begin{array}{l}\text { População de idosos com hipoalbu } \\
\text { minemia }(n=151)\end{array}$} \\
\hline Doenças $\mathrm{N}$ & Número de casos & Doenças Nú & lúmero de casos \\
\hline Pneumonia & 48 & ITU & 31 \\
\hline ITU & 46 & Pneumonia & 29 \\
\hline Neoplasia & 42 & Neoplasia & 27 \\
\hline $\begin{array}{l}\text { Distúrbios } \\
\text { Gástricos }\end{array}$ & 40 & $\begin{array}{l}\text { Distúrbios } \\
\text { Gástricos }\end{array}$ & 26 \\
\hline Cardiopatias & 35 & Cardiopatias & 18 \\
\hline AVE & 32 & DM & 14 \\
\hline DM & 22 & AVE & 14 \\
\hline DPOC & 22 & DPOC & 8 \\
\hline
\end{tabular}

DPOC = doença pulmonar obstrutiva crônica; ITU = infecção do trato urinário

Medicamentos prescritos

Neste estudo foram encontrados 187 diferentes medicamentos prescritos, totalizando 204 princípios ativos. A média de medicamentos prescritos durante o período de internação foi de $6,46 \pm 3,67$.

Os 20 medicamentos mais frequentemente utilizados entre os idosos com hipoalbuminemia, que representam conjuntamente $67,9 \%$ de todos os medicamentos prescritos, foram distribuídos em 12 classes terapêuticas e 19 apresentaram afinidade à albumina.

Ao analisar as classes terapêuticas e os medicamentos mais prescritos aos pacientes com hipoalbuminemia, encontramos os antiulcerosos $(18,2 \%)$ sendo representados pelo cloridrato de ranitidina e omeprazol; os antibióticos (16,9\%), representados pelo ceftriaxona, levofloxacino, ampicilina sódica associada ao subactam e ciprofloxacino; os diuréticos (15,8\%) furosemida, espironolactona e hidroclorotiazida; os anti-hipertensivos $(12,5 \%)$ captopril e nifedipino; o anticoagulante enoxaparina sódica (9,8\%); os cardiotônicos (5,7\%) deslanósido e digoxina; o analgésico dipirona sódica (5,1\%); o antiemético metoclopramida (4,4\%); o ansiolítico diazepan (3,9\%); o antiagregante plaquetário ácido acetilsalicílico (3\%); o anti-inflamatório corticosteroide hidrocortisona $(2,4 \%)$ e o antiarrítmico amiodarona $(2,2 \%)$ (Tabela 2).

As vias de administração mais utilizadas por este grupo foram a endovenosa (45,5\%), seguida da oral (44\%), via subcutânea $(6,6 \%)$ sonda nasoenteral e nasogástrica $(2,6 \%)$, intramuscular $(1,2 \%)$ e sublingual $(0,1 \%)$.

Não foram observadas diferenças significativas com relação ao número de medicamentos com afinidade à albumina administrada aos pacientes com hipoalbuminemia e com albuminemia normal $[p=0,26]$.

$\mathrm{Na}$ população com hipoalbuminemia, observou-se que $30,5 \%(n=46)$ deles fizeram uso exclusivo de medicamentos com afinidade à albumina.

Entre os 151 idosos com hipoalbuminemia 57,6\% $(n=87)$, fizeram uso de quatro a sete diferentes medicamentos, enquanto

\begin{tabular}{|c|c|c|c|}
\hline $\begin{array}{l}\text { Classe } \\
\text { Terapêutica }\end{array}$ & Medicamento & $\begin{array}{c}\text { Frequência } \\
\text { de prescrição }\end{array}$ & $\begin{array}{l}\text { \% ligação à } \\
\text { albumina }\end{array}$ \\
\hline \multirow[t]{2}{*}{ Antiulcerosos } & $\begin{array}{l}\text { Cloridrato de } \\
\text { ranitidina }\end{array}$ & 97 & 30 \\
\hline & Omeprazol & 24 & 95 \\
\hline \multirow[t]{4}{*}{ Antibióticos } & Ceftriaxona & 33 & 85 \\
\hline & Levofloxacino & 30 & 30 \\
\hline & $\begin{array}{c}\text { Ampicilina sódica }+ \\
\text { Subactam }\end{array}$ & 27 & 20 \\
\hline & Ciprofloxacino & 22 & 30 \\
\hline \multirow[t]{3}{*}{ Diuréticos } & Furosemida & 65 & 97 \\
\hline & Espironolactona & 27 & 90 \\
\hline & Hidroclorotiazida & 13 & 40 \\
\hline \multirow[t]{2}{*}{ Anti-hipertensivos } & Captopril & 64 & 30 \\
\hline & Nifedipino & 19 & 98 \\
\hline $\begin{array}{l}\text { Anticoagulante e } \\
\text { antitrombótico }\end{array}$ & Enoxaparina Sódica & 65 & $\begin{array}{l}\text { Índice não } \\
\text { encontrado }\end{array}$ \\
\hline \multirow[t]{2}{*}{ Cardiotônicos } & Deslanósido & 20 & 25 \\
\hline & Digoxina & 18 & 25 \\
\hline $\begin{array}{l}\text { Analgésico e } \\
\text { Antipirético }\end{array}$ & Dipirona Sódica & 34 & 58 \\
\hline Antiemético & Metoclopramida & 29 & 32 \\
\hline Ansiolítico & Diazepan & 26 & 98 \\
\hline $\begin{array}{l}\text { Antiagregante } \\
\text { plaquetário }\end{array}$ & Ácido Acetilsalicílico & 20 & 90 \\
\hline $\begin{array}{l}\text { Anti-inflamatório } \\
\text { Corticosteroide }\end{array}$ & Hidrocortisona & 16 & 99 \\
\hline Antiarrítmico & Amiodarona & 15 & 98 \\
\hline
\end{tabular}

$9,9 \%(n=15)$ utilizaram de oito a 16 medicamentos, todos com afinidade à albumina.

Não foi considerada a repetição de um mesmo medicamento para o mesmo paciente.

Período de internação e fatores influenciadores do desfecho clínico

Dos 277 pacientes investigados, $86,6 \%$ obtiveram alta, enquanto que 13,4\% foram a óbito (Tabela 3). Sendo que destes, 20 pacientes eram do sexo feminino e 17 do sexo masculino. 0 período médio de internação da população total de idosos foi de $16 \pm 12$ dias, sendo que aqueles com hipoalbuminemia apresentaram um período de internação de $18,49 \pm 14,16$ dias, enquanto aqueles com nível de albumina normal tiveram permanência média de internação de $17,5 \pm 10,97$ dias. Com relação ao período de internação, não foram encontradas diferenças significativas entre os pacientes com hipoalbuminemia e com níveis de albumina normal $[p=0,73]$.

No entanto, vale ressaltar que o período de internação não apresentou diferença significativa quando avaliada por grupos, ou seja, pacientes com hipoalbuminemia que obtiveram alta vs pacientes com hipoalbuminemia que evoluíram ao óbito $[p=0,55]$. Entretanto, a relevância desta análise não pode ser considerada devido a magnitude do desvio padrão, que demonstrou não haver homogeneidade interna nos grupos. Por se tratar de uma análise de 
variância, a homogeneidade intragrupo é um pressuposto essencial.

No entanto, todos os pacientes com índices normais de albuminemia tiveram alta hospitalar, enquanto que todos os que foram a óbito possuíam hipoalbuminemia. Ainda, entre aqueles com hipoalbuminemia 20,5\% evoluíram ao óbito e 79,5\% obtiveram alta (Tabela 3). Constatou-se diferença significativa entre os níveis de albuminemia no grupo de pacientes com hipoalbuminemia quando comparados àqueles que foram ao óbito $(2,40 \pm 0,47 \mathrm{~g} / \mathrm{dL})$ e os que obtiveram alta $(3.0 \pm 0,52 \mathrm{~g} / \mathrm{dL})\left(\mathrm{t}_{(177)}=-5.82 ; \mathrm{p}=0,001\right)$.

Diferenças significativas foram encontradas quanto ao número de medicamentos com afinidade à albumina utilizados nos grupos de pacientes com hipoalbuminemia que receberam alta e nos que culminaram em óbito [t(149) =-2,419; $p=0,02]$. Aqueles que faleceram consumiam maior número desses fármacos ( $6 \pm 2,44$ medicamentos) em contraposição aos que receberam alta ( $5 \pm 2,89$ medicamentos) (Tabela 3 ).

Tabela 3 - Distribuição dos idosos quanto aos níveis de albumina sérica, administração de medicamentos com afinidade a albumina e condição de saída hospitalar

\begin{tabular}{lccccc}
\hline \multirow{2}{*}{$\begin{array}{l}\text { Níveis de } \\
\text { albuminemia }\end{array}$} & \multirow{2}{*}{ Amostra } & \multicolumn{2}{c}{ Média de medicamentos } & \multicolumn{2}{c}{$\begin{array}{c}\text { Condição de } \\
\text { saída hospitalar }\end{array}$} \\
\cline { 3 - 6 } & & Alta & Óbito & Alta & Óbito \\
Hipoalbuminemia & 151 & $5 \pm 2,89$ & $6 \pm 2,44$ & 120 & 31 \\
Não mensurada & 98 & $4,05 \pm 2,27$ & $2,83 \pm 1,16$ & 92 & 6 \\
Albumina normal & 28 & $4,35 \pm 2,16$ & 0 & 28 & 0 \\
Total & 277 & - & - & 240 & 37 \\
\hline
\end{tabular}

Deve-se ressaltar que a média de medicamentos utilizados pelos pacientes do grupo com hipoalbuminemia (óbito e alta) e o grupo de albuminemia normal apresentaram diferença significativa $\left(t_{(177)}=2.66 ; p=0,008\right)$. Em adição, dos 31 pacientes com hipoalbuminemia que foram ao óbito, 30 faziam uso de três ou mais medicamentos com afinidade à albumina e seis fizeram uso exclusivo de medicamentos com afinidade a esta proteína.

Quando se avaliou a hipótese do sexo, idade, dias de internação, número de medicamentos com e sem afinidade à albumina, à albuminemia e número de doenças motivadoras da internação enquanto preditores do óbito observou-se que o número de medicamentos com afinidade à albumina, à albuminemia e o período de internação foram às variáveis preditoras do óbito $\left(r=0,47 ; R^{2}=0,224\right),[F(8.170)=6.13 ; p=0.001]$.

A relação entre a variável dependente e as independentes resultou na equação matemática: [óbito $=0,545$ - 0,374 (albuminemia)- 0,195 (dias de internação) 0,175 (número de medicamentos com afinidade à albumina)].

\section{Discussão}

A pesquisa demonstrou que a maioria dos idosos investigados quanto aos níveis de albumina sérica apresentaram hipoalbuminemia, porcentual que pode estar subestimado já que para parte da população investigada não foi solicitado os níveis séricos desta proteína, apesar de estudos evidenciarem o nível sérico de albumina como preditor de risco de morte ${ }^{15,16}$.
A hipoalbuminemia pode apontar para um perfil de desnutrição nosocomial, e/ou ser um indicador de atividade inflamatória, ,5,6,7 e/ou ainda um importante fator de risco para o desenvolvimento de complicações infecciosas pós- trauma ${ }^{5}$. Cooper et al. também associaram os baixos níveis de albumina plasmática a doenças vasculares ${ }^{24}$.

A questão relacionada à desnutrição intra-hospitalar vem sendo referenciada há alguns anos pelo Inquérito Brasileiro de Avaliação Nutricional Hospitalar (IBRANUTRI) realizado em 1996. Naquele estudo, foi revelado um elevado porcentual $(48,1 \%)$ de desnutrição intra-hospitalar nos hospitais públicos e a dosagem de albumina sérica foi investigado em 23,5\% dos casos, o que corrobora com os resultados obtidos na nossa pesquisa ${ }^{18}$

Nossos resultados demonstraram uma média alta de medicamentos prescritos, assim como encontrado em outras pesquisas, ${ }^{10,11,12,25}$ sendo significativo o consumo de fármacos entre os idosos, em especial os com alta afinidade pela albumina $^{4,26}$. Entretanto, a inobservância, no que tange à prescrição destes medicamentos, a pacientes com hipoalbuminemia foi constatada pelo presente trabalho, sendo que os riscos de se desenvolver reações adversas pela inadequada prescrição de medicamentos a idosos estão descritos na literatura ${ }^{2,27,28}$.

As principais classes terapêuticas utilizadas foram os antihipertensivos e diuréticos, cuja prevalência justifica-se pela importante presença da hipertensão arterial sistêmica neste estudo assim como na população idosa brasileira29,30,31. O elevado índice de antibióticos prescritos resulta provavelmente do fato de doenças infecciosas estarem presentes entre a população estudada. Este achado está em consonância com outros trabalhos publicados, que mostram a prevalência de doenças infecciosas entre os idosos, inclusive por pneumonias ${ }^{18,32,33,34}$, sendo, sobretudo, relacionada à alta frequência de mortalidade, especialmente em quadros de desnutrição ${ }^{35,36}$.

Quanto aos fatores influenciadores do prognóstico de idosos participantes da pesquisa, verificou-se uma diferença significativa nos níveis de albumina entre os pacientes com hipoalbuminemia que faleceram e que obtiveram alta. Aqueles pacientes que foram ao óbito mostraram níveis de albuminemia menores, ressaltando a relevância deste parâmetro sobre o desfecho clínico.

A respeito dos idosos com hipoalbuminemia em uso de grande quantidade de medicamentos com afinidade à albumina, pode-se inferir que ocorreram modificações farmacocinéticas e farmacodinâmicas importantes, cujas consequências não puderam ser avaliadas em nosso estudo. A relevância clínica da competição entre as moléculas de fármacos pelos sítios de ligação a esta proteína podem comprometer o sucesso da farmacoterapia. Uma vez que pode ocorrer aumento do efeito inicial, evidenciando toxicidade de alguns fármacos e alterações em sua meia vida plasmática ${ }^{37}$.

A literatura aponta que a concentração de proteínas séricas deve ser monitorada para contribuir com eficácia e segurança na utilização de terapia medicamentosa com fármacos que apresentam afinidade por estas proteínas ${ }^{38}$. Apesar da hipoalbuminemia ser um distúrbio nutricional frequentemente relatado em pesquisas clínicas ${ }^{9,39}$ e muitas enfermidades compartilharem a responsabilidade pela hipoalbuminemia, é possível constatar 
que o monitoramento dos níveis séricos desta proteína não se constituiu em rotina na conduta clínica, ainda que na presença de doenças indutoras destes distúrbios.

O desenvolvimento e a implementação de protocolos para a prescrição terapêutica em virtude das especificidades farmacocinéticas na senescência e do estado nutricional e de saúde dos idosos é outro aspecto a ser considerado.

Os resultados obtidos em nossa pesquisa demonstraram que os valores de albuminemia e os dias de internação estão inversamente correlacionados ao óbito e diretamente correlacionados ao elevado número de medicamentos ligados à albumina. Estas correlações podem estar sinalizando que as variáveis analisadas podem contribuir para o aumento das comorbidades e mortalidade intra-hospitalar em idosos.

A utilização elevada do número de medicamentos em idosos, em especial em ambiente nosocomial, se constitui em preocupação em decorrente dos possíveis resultados negativos advindos de interações medicamentosas, reações adversas e outros. No entanto, torna-se relevante perceber que a associação observada com o óbito se deu em função do número de medicamentos com afinidade a albumina e não ao número total de medicamentos utilizados.

Embora tivéssemos verificado o número de doenças apresentadas pelo paciente, por se tratar de uma pesquisa retrospectiva, não foi possível acompanhar algumas outras variáveis que poderiam influenciar o óbito, tal como a gravidade da doença, o que representa uma limitação do nosso estudo. Por outro lado, pacientes com risco iminente de óbito costumam encontrarem-se internados em Unidade de Terapia Intensiva, o que não correspondia a unidade investigada neste trabalho. Desta forma, a associação de múltiplos medicamentos ligados a albumina e administrados a pacientes com hipoalbuminemia, podem ter contribuído para o agravamento da doença, desfecho clínico desfavorável e consequente redução do período de internação.

\section{Conclusão}

Dentre os fatores investigados a hipoalbuminemia, o tempo de internação e o número de medicamentos prescritos que possuíam afinidade pela albumina apresentaram associação com o prognóstico desfavorável dos idosos alvos deste estudo, merecendo, portanto, serem monitorados atentamente pelos profissionais de saúde.

\section{Suporte Financeiro:}

Este trabalho foi realizado sem ônus para a instituição e sem apoio financeiro.

\section{Conflito de interesse: não há}

\section{SUMMARY}

Albumin AND DRUG therapy in the PROGNOSIS OF hospitalized ELDERLY

OBJECTIVE. This study aimed to investigate the relationship between serum albumin levels, drugs used, length of hospitalization and number of diseases with the prognosis of hospitalized elderly.
Methods. A descriptive cross-sectional and retrospective study was carried out using a review of medical charts. Variables collected were disease motivating admission, albumin, drugs used and their affinity for albumin and length of stay in an elderly population of a Brazilian public hospital. We used multiple regression to assess whether age, length of stay, medication with or without affinity to albumin and number of drugs motivating admission were causes of death. The relation between dependent and independent variables was analyzed by two-way ANOVA.

RESULTS. The sample consisted of 277 elderly, 151 with hypoalbuminemia and those using an average of $6.6 \pm 3.80$ different prescription drugs most of which had affinity for albumin (4.96 \pm 2.64$)$. Results showed that the number of drugs with affinity for albumin and hospital stay were predictors of death $(r=0.47, R 2=0.224),(F(8.170)=6.13$; $p=0.001)$. The relation between dependent and independent variables resulted in the mathematical equation: (death= $0.545-0.374$ (albumin) - 0.195 (days of hospitalization) + 0.175 (number of drugs with affinity for albumin)).

CONCLUSION. The mathematical equation obtained by this study demonstrated that albumin and days of hospitalization were inversely correlated with death and directly correlated with the number of drugs bound to albumin. [Rev Assoc Med Bras 2010; 56(2): 145-50]

KEY WORDS: Frail elderly. Hypoalbuminemia. Prescription drugs.

\section{REFERÊNCIAS}

1. Carvalho JAM, Garcia RA. The aging process in the Brazilian population: a demographic approach. Cad Saúde Pública. 2003;19(3):725-33.

2. Lima-Costa MF, Veras R. Saúde pública e envelhecimento. Cad Saúde Pública. 2003; 19(3):700-1.

3. Filho JMC, Marcopito LF, Castelo A. Perfil de utilização de medicamentos por idosos em área urbana do Nordeste do Brasil. Rev Saúde Pública. 2004;38(4):557-64.

4. Camarano AA, Beltrão KL, Pescon ARP, Medeiros M, Carneiro IG, Goldoni AM, et al. Como vai o idoso brasileiro? Rio de Janeiro: IPEA; 1999. Texto para discussão $n^{\circ} 681$.

5. Soeters PB, Reijven PLM, Bokhorst-de van der Schueren MAE, Schols JMGA, Halfens RJG, Meijers JMM, et al. A rational approach to nutritional assessment. Elsevier Ltd and European Society for Clinical Nutrition and Metabolism. Clin Nut. 2008;(27):706-16.

6. Johnson AM. Low Levels of plasma proteins: malnutritionn or inflammation? Clin Chem Lab Med. 1999;37(2):91-6.

7. Cueto MAM. Hypoalbuminemia in dialysis. Is it a marker for malnutrition or inflammation? Rev Invest Clin. 2001; 53(2):152-8.

8. Waitzberg DL, Gama-Rodrigues J, Correia NITD. Desnutrição hospitalar no Brasil. In: Waitzberg DL. Nutrição oral, enteral e parenteral na prática clínica. $3^{a}$ ed. São Paulo: Atheneu; 2000. v. 1, p. 385-95.

9. Waitzberg DL, Caiaffa WT, Correia MITD: Inquérito Brasileiro de Avaliação Nutricional Hospitalar (IBRANUTRI). Rev Bras Nutr Clin. 1999;114(1):123-33.

10. Silva P, Luis S, Biscaia A. Polimedicação: um estudo de prevalência nos Centros de Saúde do Lumiar e de Queluz. Rev Port Clin Geral. 2004;20(3):323-36.

11. Teixeira JJV, Lefèvre F. A prescrição medicamentosa sob a ótica do paciente idoso. Rev Saúde Pública. 2001;35(2):207-13.

12. Locatelli J. Drug interactions in hospitalized elderly patients. Einstein. 2007;5(4):343-6.

13. Roughead EE, Gilbert AL, Primrose JG, Sansom LN. Drug-related hospital admissions: a review of Australian studies published 1988-1996. Med J Aust. 1998;168(8):405-8.

14. Schneider JK, Mion LC, Frengley JD. Adverse drug reactions in an elderly outpatient population. Am J Hosp Pharm. 1992; 49(1):90-6.

15. Gibbs J, Cull W, Henderson W, Daley J, Hur K, Khuri SF. Preoperative serum albumin level as a predictor of operative mortality and morbidity: results from the National VA Surgical Risk Study. Arch Surg. 1999; 134(1):36-42.

16. Goldwasser P, Feldman J. Association of serum albumin and mortality risk. J Clin Epidemiol. 1997; 50(6):693-703. 
17. DATASUS. Departamento de Informação e Informática do SUS. Indicadores e dados básicos. Brasil: DATASUS. [citado 20 nov 2008]. Disponível em: http://www.datasus.gov.br/datasus/datasus.php.

18. Paes NA. A mortalidade por doenças infecciosas e parasitárias na população idosa brasileira. Rev Panam Salud Publica. 2004;15(4):233-41.

19. World Health Organization/WHO Collaborating Centre for Drug Statistics Methodology. Anatomical therapeutic chemical (ATC) classification index-including defined daily doses (DDDs) for plain substances. Oslo: WHO-Oslo, 2008. [cited 2008 oct]. Available from: http://www.whocc. no/atcddd.

20. Micromedex ${ }^{\circledR}$ Healthcare Series. [citado out 2008]. Disponível em: http:// www.thomsonhc/librarian/PFDefaultActionld/hsc. Interactions. Worldheel.

21. Organización Farmacéutica Colegial. Base de datos del medicamento do Consejo General de Colegios Oficiales de Farmacéuticos. [citado out 2008]. Disponível em: http://www.pfarmals.portalfarma.com/defalt.asp.

22. Cuppari L. Guias de medicina ambulatorial e hospitalar - UNIFESP / Escola Paulista de Medicina. Nutrição clínica no adulto. São Paulo: Manole; 2002.

23. Alcock NW. Pruebas de laboratorio para valorar el estado nutricional. En: Nutrición en Salud y Enfermedad. Shils ME, Olson JA, Shike M, Ross CA, editores. México: McGraw.Hill Interamericana; 2002. p.1057-71.

24. Cooper BA, Penne EL, Bartlett LH, Pollock CA. Protein malnutrition and hypoalbuminemia as predictors of vascular events and mortality in ESRD. Am J Kidney Dis. 2004;43(1):61-6.

25. Flores LM, Mengue SS. Uso de medicamentos por idosos em região do Sul do Brasil. Rev Saúde Pública. 2005;39(6): 924-9.

26. Schmidt S, Rock K, Sahre M, Burkhardt O, Brunner M, Lobmeyer MT, et al. Effect of protein binding on the pharmacological activity of highly bound antibiotics. Antimicrob Agents Chemother. 2008;52(11):3994-4000.

27. Ribeiro AQ, Araújo CMC, Acurcio FA, Magalhães SMS, Chaimowicz F. Quality assessment of drug use in the elderly: a review of available evaluation methods. Ciência \& Saúde Coletiva. 2005;10(4):1037-45.

28. Heller TA, Larson EB, LoGerfo JP. Quality of ambulatory care of the elderly: an analysis of five conditions. J Am Geriatr Soc.1984;32(11):782-8.

29. Mosegui GBG, Rozenfeld S, Veras RP, Vianna CMM. Avaliação da qualidade do uso de medicamentos em idosos. Rev. Saúde Pública. 1999;33(5):437-44.

30. Barreto MS, Passos VMA, Firmo JOA, Guerra HL, Vidigal PG, Lima-Costa MF. Hypertension and clustering of cardiovascular risk factors in a community in southeast Brazil - the Bambuí Health and ageing study. Arq Bras Cardiol. 2001;77(6): 576-81.

31. Marin MJS, Cecílio LCO, Perez AEWUF, Santella F, Silva CBA, Filho JRG, et al. Caracterização do uso de medicamentos entre idosos de uma unidade do Programa Saúde da Família. Cad Saúde Pública. 2008;24(7):1545-55.

32. Almeida JR, Filho OFF. Pneumonias adquiridas na comunidade em pacientes idosos: aderência ao Consenso Brasileiro sobre Pneumonias. J Bras Pneumol. 2004; 30(3):229-36.

33. Villas Bôas PJF, Ferreira ALA. Infecção em idosos internados em instituição de longa permanência. Rev Assoc Med Bras. 2007;53(2):126-9.

34. Chong CP, Street PR. Pneumonia in the elderly: A review of severity assessment, prognosis, mortality, prevention, and treatment. South Med J. 2008;101(11):1084-5.

35. Gee Mac W. Causes of death in a hospitalized geriatric population: an autopsy study of 3000 patients. Virch Archiv. 1993;423(5):343-9.

36. Sullivan DH, Sun S, Walls RC. Protein-energy undernutrition among elderly hospitalized patients. JAMA. 1999;281(21):2013-9.

37. Silva, P. Farmacologia. $5^{a}$ ed. Rio de Janeiro: Guanabara Koogan; 1998. p. 58.

38. Mônica KG, Douglas BF. Age-related changes in protein binding of drugs: implications for therapy. Clin Pharmacokinet. 2000;38(3):271-90.

39. Sullivam DH, Roberson PK, Bopp MM. Hypoalbuminemia 3 months after hospital discharge: significance for long-term survival. J Am Geriatr Soc. 2005;53(7):1222-6.
Artigo recebido: 08/09/09 Aceito para publicação: 20/01/00 